\title{
Author Index to Volume 21
}

Ackerman NB, 594

Agata $Y, 590$

Alverson D, 447

Ambruso DR, 205

Ament ME, 399, 538

Anderson S, 99

Andersson S, 396

Annerén KG, 88

Aprille JR, 266

Arab N, 257

Arima M, 34

Armengol J, 183

Armstrong MJ, 261

Atkinson M, 104

Augustine NH, 517

Aw TY, 492

Bachmann C, 201

Badura RJ, 121

Baker SB, 116

Baley JE, 306

Balfe JW, 296

Balistreri WF, 417

Ballard PL, 142

Ballke EH, 283

Barrington KJ, 166, 247

Bartal L, 54

Batton DG, 511

Baumgarten L, 568

Beasley DG, 188, 534

Beemer FA, 502

Beitins IZ, 409

Berger M, 306

Berger R, 279

Berman W Jr, 447

Biggs DE, 409

Bijman J, 79

Blades BL, 608

Boroujerdi MA, 10

Bourbon J, 436

Bradt SK, 14

Brady JP, 313

Braggion M, 462

Branski D, 414

Bratton D, 159

Breidenstein J, 197

Breslin JS, 417

Briggs RW, 357

Brohn F, 275

Brubakk A-M, 29

Bruce MC, 306

Bruinvis L, 502

Bruneau M, 579

Brun P, 362

Burdick B, 152

Busija DW, 188, 534

Callegari CC, 453, 590

Carlson SE, 507

Cha C-JM, 83

Chang B, 104

Cheung ATW, 170

Chevalier RL, 338

Cleary ML, 331

Coalson JJ, 594

Coen P, 58

Cohen MB, 551

Cole DEC, 296

Collins JE, 10

Colombo C, 197
Colombo J-P, 201

Connor EM, 547

Cooper DM, 568

Courtney SE, 381

Cowan BE, 357

Cox KL, 170

Daffos F, 579

Dannan G, 257

Dattel BJ, 142

Davis DJ, 142

Delemos RA, 590

Delvin EE, 432

Derr JA, 66

Desai N, 371

Desjeux JF, 477

Diver-Haber A, 126

Douglas SD, 542

Dudgeon DL, 422

Dudman NPB, 608

Dumaswala R, 417

Dumontier AM, 477

Dunn LL, 530

Dupret JM, 362

Duran M, 137, 502

Engelhardt B, 159

Ennever JF, 530

Epstein CJ, 88

Epstein LG, 547

Eran M, 414

Escobedo MB, 594

Eyal FG, 183

Facchin P, 462

Falace P, 371

Faucher DJ, 38

Felder CC, 497

Feltes TF, 131

Fernandes J, 279

Ferrante A, 377

Fewell JE, 116

Filler RM, 242

Finer NN, 166, 247

Fischer GW, 326

Fisher DA, 453

Fisher SE, 104

Fletcher AM, 534

Fong JSC, 252

Forestier F, 579

Francalancia NA, 58

Frank L, 109, 427

Freedman J, 152

Freier S, 414

Fritchman K, 371

Fyhrquist F, 396

Gahl WA, 193

Galili N, 331

Gallo RC, 547

Garfield SA, 417

Gartner LM, 487

Garza C, 301

Gelardi NL, 83

Geoffray DJ, 391

Gerdes JS, 542

Gerrard JM, 293

Gerstmann DR, 594

Ghishan FK, 257, 404
Giannella RA, 551

Gibson BE, 205

Giesler ME, 131

Gitzelmann R, 201

Golden SM, 326

Goldgar DE, 507

Goldman SL, 313

Gouyon J-B, 615

Gowen B, 293

Grand RJ, 261

Greenberg JW, 530

Greene HL, 404

Green RS, 534

Grogaard J, 159

Gross-Kieselstein E, 414

Gruber WC, 270

Grundberg CM, 296

Guarino A, 551

Guignard J-P, 615

Gustafsson J, 99

Guzick DS, 386

Haddad GG, 556

Halvorsen S, 1, 148

Hamilton SR, 422

Hankins GDV, 386

Hanning RM, 296

Hansen TN, 131

Harris MC, 542

Hartman AD, 391

Hayashi A, 34

Hayek Z, 183

Hazen-Martin DJ, 72, 235

Heim T, 242

Hellerqvist C, 159

Hemming VG, 326

Hennigar RA, 235

Henry JF, 563

Heymann MA, 176

Hill HR, 517

Hirose S, 21

Holter PH, 1

Hopson JF, 381

Houser MT, 442

Hynynen M, 396

Israels SJ, 293

Iwahashi-Hosoda CK, 170

Iwai Y, 285

Jaehrig K, 283

Jeng HJ, 556

Jenkins MQ, 72

Johnson RM, 275

Jonas A, 126

Jones CE, 338

Jones DP, 492

Jones PJH, 242

Jones R, 183

Jose PA, 497

Kaiser DL, 338

Kanto WP Jr, 368

Kaplan B, 126

Kaplan BS, 252

Kaplan MR, 568

Karp WB, 368

Keelan M, 347

Kerrigan JR, 58
Ketting D, 502

Kikuchi K, 257

Kim KS, 289

Kimura RE, 214

Kirby ML, 219

Klein AH, 453

Klück P, 466

Koenig A, 283

Kohlman L, 352

Kojima T, 34

Kopple JD, 399

Kowanko IC, 377

Ktorza A, 436

Kuehl TJ, 594

Kuis W, 137

LaFramboise WA, 238

Laidlaw SA, 399

Lai TL, 556

Laptook AR, 38

Larroya S, 352

Leblanc AL, 131

Lee J-C, 542

Leffler CW, 188, 534

Legrain M, 477

Lemons JA, 44

Leonard JV, 10, 211

Lewis DE, 331

Liechty EA, 44

Liggins GC, 603

Lipson M, 371

Lister G Jr, 447

Liu YA, 296

Lohse CL, 170

Lowe TW, 38

Lu D, 357

Lyons DT, 471

MacDonald PC, 386

Magness RR, 38

Malhotra V, 530

Marin L, 436

Marks KH, 58, 66

Martin CG, 131

Matherne GP, 229

Mathieu H, 362

Maxwell GM, 377, 573

Maybee DA, 326

Mayfield SR, 93, 482

Mayock DE, 25, 121, 238

Mayumi M, 285

McCann EM, 313

McFall TL, 517

McKee KS, 563

McNellis W, 49

McPhee AJ, 573

McWeeny OJ, 229

Medvik KA, 306

Meijers JHC, 466

Meisel P, 283

Mellins RB, 556

Meretoja O, 396

Michael L, 152

Middleton B, 211

Mikawa H, 285

Miller HI, 391

Miller T, 152

Minnefor AB, 547

Mitsudome A, 21

Miyanomae T, 285 
Molenaar JC, 466

Montgomery RK, 261

Moon A, 371

Moore MC, 404

Morin FC III, 225

Morrison A, 352

Moscioni AD, 487

Muramatsu K, 21

Nakajima $\mathrm{H}, 34$

Nakamoto T, 391

Nakamura KT, 229

Nardis EE, 58, 66, 511

Newnham J, 453

Nichols BL, 563

Nissim I, 49

Nosek MT, 266

Nose O, 538

Nosko M, 166

Null DM Jr, 594

O'Brien WF, 326

Oda $T, 21$

Ogata $\mathrm{H}, 21$

Oh W, 29, 83, 93, 482

Oleske JM, 547

Olsen S, 447

Olson TA, 326

O'Neill D, 14

Oren M, 126

Orth DN, 404

Padbury JF, 453, 590

Padeh S, 54

Pagnan A, 462

Parikh N, 331

Parker CR Jr, 386

Passwell J, 126

Passwell JH, 54

Pasternack G, 235

Pavanello L, 462

Peeters LL, 584

Peliowski A, 166

Perez A, 261

Perret C, 362

Pesonen E, 396

Pignol B, 436

Pilon A-M, 432

Polak MJ, 44

Polin R, 49
Polin RA, 542

Polk DH, 453, 590

Porter JC, 38

Putet G, 458

Putman M, 563

Quinton PM, 79

Rainaut M, 579

Rao VS, 507

Ravindranath Y, 275

Refsum HE, 1

Reiter EO, 409

Reviczky A, 453

Rhead WJ, 371

Rhodes PG, 507

Rietort M, 436

Rigo J, 458

Rijksen G, 137

Ritz J, 331

Robert-Guroff M, 547

Roberts JM, 142

Robertson AF, 368

Robillard JE, 229, 497

Robotham JL, 594

Ronchi M, 197

Rosenblatt HM, 331

Rosenfeld CR, 38, 386

Ross B, 152

Roy S III, 497

Ryan CA, 166

Said HM, 404

Salle B, 458

Sandberg K, 159

Sanengen T, 1, 148

Saxena PR, 584

Schanler RJ, 301

Schellenberg J-C, 603

Schneider PA, 422

Schoeller DA, 242

Schreiber MD, 176

Schwarz KB, 352

Seakins JWT, 211

Seidel CL, 152

Sena M, 357

Sens DA, 72, 235

Sens MA, 72

Senterre J, 458

Setchell KDR, 197, 417
Shaffer SG, 14

Sharon P, 414

Shaul PW, 482

Shearer WT, 331

Sidran MP, 462

Siervogel RM, 381

Silbajoris R, 5

Simell O, 477

Sjövall J, 99

Sklar J, 331

Smith BA, 229

Smith J, 242

Smith S, 131

Snider MJ, 58

Soifer SJ, 176

Sonksen PH, 10

Sosenko IRS, 427

Spaapen LJM, 137

Spain CL, 5

Spicer SS, 72, 235

Staal GEJ, 137

Standaert TA, 25, 121, 238

Steinmann B, 201

Stewart AW, 603

Stonestreet BS, 29, 93, 482

Stoop JW, 137

Stork LC, 205

Sturgill BC, 338

Suchy FJ, 417

Sugita K, 34

Sundell H, 159

Suzuki N, 34

Sweetman L, 371

Swyer PR, 242

Szabo JS, 93

Tanabe Y, 34

Tanaka M, 285

Tavernini M, 347

Temples TE, 391

Thibeault DW, 14

Thomasset M, 362

Thomson ABR, 347

Throop BJ, 270

Thurman GW, 205

Tibboel D, 466

Tietze F, 193

Tikkanen I, 396

Tipton JR, 538

Tordet C, 436

Trivin F, 579
Umpleby AM, 10

Van Den Abbeele A, 159

van der Kamp AWM, 466

Van Haperen-Heuts ICCM, 466

Vannucci RC, 471, 524

Vannucci SJ, 524

Vasta F, 471, 524

Vedanarayanan VV, 252

Vekemans M, 432

Veldhuis JD, 409

Verkeste CM, 584

Vinton NE, 399

Vonlanthen M, 477

Voorbrood BS, 137

Wachtl JP, 381

Wadman SK, 137, 502

Wallenburg HCS, 584

Walsh EM, 170

Wang DL, 590

Wasserman K, 568

Watchko JF, 25, 121, 238

Weidenfeld J, 414

Weiler-Ravell D, 568

Westphal MC, 72

Whipp BJ, 568

Whitington PF, 487

Wilcken DEL, 608

Wilkinson MH, 247

Wilson SZ, 270

Winthrop AL, 242

Wolff JA, 371

Woodrum DE, 25, 121, 238

Wyde PR, 270

Yabuuchi H, 538

Yoshimura T, 285

Young RSK, 357

Young SL, 5

Yudkoff M, 49

Yukitake K, 21

Zacchello G, 462

Zegers BJM, 137

Ziegler MM, 542

Zimmerman GA, 517

Ziron L, 462

Zlotkin SH, 296

Zuliani G, 197 